\title{
Psychosocial Impact of Night Shift Work among Nurses in Saudi Arabia
}

\author{
Abdalkarem F. Alsharari* \\ Nursing Department, College of Applied Medical Sciences, Jouf University, Saudi Arabia \\ *Corresponding author: afalsharari@ju.edu.sa \\ Received January 04, 2019; Revised February 11, 2019; Accepted March 04, 2019
}

\begin{abstract}
Background: A considerable number of studies have identified psychological and social difficulties resulting from night shift work among nurses. However, studies of the risk factors of experiencing the psychological and social impact of night shift work among hospital nurses is crucial but lacking. This study aimed to investigate the psychological and social effects of night shift work and the associated factors among nurses in Saudi Arabia (SA). Methods: This was a cross-sectional, descriptive survey carried out from May to July 2017 among nurses working night shifts in public hospitals in all regions of SA. The data were collected through online or paper-based self-administered questionnaires. Multivariable logistic regression analysis was utilized to identify the predictors of experiencing the psychological and social impact of night shift work. Results: A total of 1521 nurses completed the survey. The overall median psychological effect score was 14.0 (maximum 20). A total of $88.2 \%(1341 / 1521)$ of the participants reported a psychological impact due to night shift work. The overall median social effect score was 15.0 (maximum 20). A total of $90.9 \%$ (1383/1521) of the participants reported a social impact due to night shift work. Being a female nurse, a Saudi or Filipino national, being involved in rotating or fixed night shift work, dislike or occasional dislike for night shift work, and having had 6-10 weeks or over 20 weeks of night shift work in the preceding year were independent predictors of having a psychological impact due to night shift work. Furthermore, being involved in rotating night shift work, dislike or occasional dislike for night shift work, and having had over 20 weeks of night shift work in the preceding year were independent predictors of having a social impact due to night shift work. Conclusion: There was a high psychosocial impact of night shift work among nurses in the study setting. The development of incentives and support structures for nurses involved in night shift work is recommended.
\end{abstract}

Keywords: psychological problems, social problems, night-shift work, self-rated health, nurses

Cite This Article: Abdalkarem F. Alsharari, "Psychosocial Impact of Night Shift Work among Nurses in Saudi Arabia." American Journal of Nursing Research, vol. 7, no. 3 (2019): 238-247. doi: 10.12691/ajnr-7-3-2.

\section{Introduction}

Globally, healthcare workers provide services to patients through shift work [1]. Shift work is an essential component of the working life for hospital nurses because nursing is a 24 hour-a-day job [1,2]. Nursing services are usually organized into three eight-hour shifts per day $[1,2]$. However, there is a trend in some settings for health service employers to adopt two longer 12-hour shifts daily [3]. Shift work that occurs during periods that differ from the traditional work schedule i.e., requiring healthcare workers to work during periods when sleep typically occurs (night shifts) and to sleep during the day substantially alters the healthcare workers' sleep-wake cycle [4]. The reversal of the sleep and wake cycles significantly distorts the healthcare workers' endogenous circadian rhythms, which results in a range of adverse consequences impacting various domains of functioning $[4,5]$. Several studies have identified night shift work as an important occupational hazard with short-term and long-term adverse effects on nurses [5-12]. In the short term, it has been found to lead to the development of physical and mental symptoms, such as burnout and exhaustion, poor quality of life, eating disorders and sleep disorders, including shift work sleep disorder [6,7,8,9]. In the long term, night shift work increases the risk of nurses becoming obese and developing impaired glucose tolerance, ischemic heart disease and cancer $[10,11,12]$. In particular, night shifts have been found to be associated with an increased risk of breast cancer development [12].

One of the major effects of night shift work on nurses is its impact on their psychological health [13,14,15]. Several studies have shown that night shifts could predispose nurses to developing mental disorders, such as anxiety and depression $[14,15]$. Other poor psychological effects associated with shift work include fatigue, mood disorders, decreased vigilance and cognitive impairment [16-19]. Thus, the psychological effects of night shifts on nurses' health can compromise their safety and the quality of the care that they provide [20]. Furthermore, night shift work can have negative impact on the emotional health, family and social life of nurses - especially for female nurses with family responsibilities (pregnancy and child raising) [21]. Moreover, beyond the hospital environment, 
night shift workers have been found to be at a higher risk of social isolation, probably due to their work schedules. Ferri et al. showed higher rates of psychological symptoms but lower rates of intimate partnership and job satisfaction among night shift nurses compared to day nurses in a study in Northern Italy [22]. In addition, Jensen et al., showed that almost a third of evening and night shift nurses in Denmark reported being socially isolated [23]. Moreover, there is a negative impact of night shift work on social engagement. In the United Kingdom (UK), an analysis of time dedicated to social participation showed that it was lower among night shift workers, including nurses [24].

In the Middle East, few studies have specifically evaluated the psychosocial impact of night shift work on nurses. In a cross-sectional study in Palestine, Jaradat et al. (2018) determined that women doing shift work had greater levels of job distress than men did, but men had less job satisfaction [25]. In Iran, a qualitative study of 18 nurses working night shifts in four hospitals identified concerns with fulfilment of duties as wives and mothers [26], while a survey of 243 female nurses in Turkey showed that work schedules and night shifts led to family conflicts and lowered life/job satisfaction [27]. In addition, a descriptive study of non-Saudi female nurses in Riyadh showed that $46.7 \%$ of nurses were overweight or obese, with nurses working night shifts reporting significantly higher levels of these conditions [28]. Self-rated assessment has been used as an indicator for all dimensions of health $[29,30]$. Studies have demonstrated that self- rated health or self- perceived health status is associated with job stress among nurses [29,30]. There is a need for a self-rating scale for the early detection of the psychological and social effects of night shift work among nurses as, while some have been used to evaluate the psychological and other effects of shift work [31,32], only a few studies have specifically investigated the associated factors. This study's objective was to investigate the psychosocial impact of night shift work and its determinants among nurses in SA.

\section{Methods}

\subsection{Study Design and Area}

This was a cross-sectional, descriptive survey carried out from May to July 2017 among nurses working night shifts in public hospitals in all 13 regions of SA. All registered nurses who undertake shift work, including night shifts in public hospitals, in the country were included, apart from those with less than one year's work experience in the nursing profession.

\subsection{Instrument}

The data were collected using a face-validated, self-administered internet/paper questionnaire designed for the purpose of the study following an extensive literature review. The questionnaire had three sections: demographics (13 questions), psychological effects of night shift work (five questions) and social effects of night shift work (five questions). The psychological effect section consisted of questions related to the nurses' attitudes, satisfaction with work schedules, feelings of depression and isolation while working night shifts, being mentally prepared for the night shift and their perception of the level of recognition accorded to nurses during the shift. Similarly, the social effect section covered questions related to the nurses being able to maintain social relationships, manage their homes and their families, family support, access to transportation while working night shifts and being able to maintain a routine social life balance. The questions consisted of positive statements that participants responded to using a 4-point Likert scale (strongly agree [1], agree [2], disagree [3] and strongly disagree [4]). A scoring system was applied, meaning that the higher the rating on the scale, the higher the psychological or social effect of the night shift work.

The study questionnaire was reviewed by a group of academics, senior nurses and public health practitioners who made minor modifications in its wording and content and who felt that the instrument had face validity. In addition, a pre-test was carried out among 20 nurses from one public hospital not used for the survey, which resulted in minor changes in the instrument.

\subsection{Data Collection}

Following the ethical approval of the study by the local Committee of Bioethics in Jouf University (6/2-37-38), the questionnaire was distributed to nurses through nursing administration in public hospitals and was made available in two formats - paper-based and electronic form. The paper-based survey was distributed among nurses in their units, where they each completed a survey at a convenient time and returned it to a special box in the nursing administration department. Nurses who preferred the electronic version of the questionnaire completed it online using their mobile phone or a computer. A QR code and a web link were also distributed among nurses using the Qualtrics platform. The questionnaire was available in the English language and a complementary Arabic version was attached for greater convenience for Arabic-speaking nurses.

The nurses were recruited using personal mobile phone and email invitations with a link to the electronic survey. Nurses were encouraged to pass on invitations to peers through personal contact and social media groups, which are common among nurses in SA, in order to maximize participation. The Qualtrics platform was utilized to collect and manage the data and included a feature to prevent multiple contributions from the same participant. No personal identifying data were collected from the nurses. All survey questionnaires contained a front explanatory page about the study's aim and purpose and the contact number of the primary investigator. Nurses who completed and returned the surveys were assumed to have given consent for participation in the study.

\subsection{Data Analysis}

The data were analyzed using SPSS version 24 (Armonk, NY: IBM Corp. USA). A composite summary score for each psychological and social effect scale was computed (minimum 5, maximum 20). The higher the 
score, the greater the impact was in each case. Participants with a score of $>10$ were considered to have psychological or social impacts in each case, while those with a score with $\leq 10$ were considered to have no psychological or social impact from night shift work. Continuous variables were summarized as means ( \pm standard deviation; SD) and median (range), while categorical variables were summarized as proportions. The normality of the totals of the psychological and social effect scores was assessed using the Shapiro-Wilk test and was found to be non-normally distributed. Categorical groups were compared using the $\chi 2$ test for proportions. The differences between the median psychological and social effect scores according to the study participants' demographic characteristics were estimated using the Mann-Whitney test and the Kruskal-Wallis test (where appropriate). Multivariable logistic regression analyses were performed to determine the factors associated with the psychological and social impact of night shift work using the full model fits. In all cases, a two-sided P-value $<0.05$ was considered statistically significant.

\section{Results}

\subsection{The Participants' Sociodemographic Profile}

A total of 1638 nurses participated in the survey, of which 1521 nurses had no missing data in their questionnaire and were retained for analysis. The participants' sociodemographic characteristics are as shown in Table 1.

The majority of the participants belonged to the 21-30 years $(55.1 \%$ - 838/1521) and 31-40 years (40.0\% $608 / 1521)$ age groups and $51.8 \%(788 / 1521)$ were males. A total of $92.4 \%(1406 / 1521)$ of the participants were Saudi nationals, $66.1 \%(1005 / 1521)$ were married and $94.9 \%$ (1443/1521) were Muslims. In addition, most of the nurses had either a diploma $(58.4 \%-888 / 1521)$ or a bachelor's degree (33.8\% - 514/1521); 53.0\% (807/1521) of the participants had one to six years work experience and $73.4 \%(1117 / 1521)$ were staff nurses. A total of $26.1 \%(397 / 1521)$ of the participants worked in the emergency unit. The study included participants from all 13 regions of SA, with Makkah $(21.7 \%$ - 330/1521) and Asser (17.0\% - 258/1521) having the highest proportion of cases (Table 1).

Furthermore, $75.1 \% \quad(1142 / 1521)$ of the nurses indicated that they were involved in rotating night shift work, where a nurse works the night shift for two or three days in a row and then changes to other work shifts during the week. The remainder worked either fixed night shifts $(10.8 \%$ - 165/1521), where a nurse is involved in continuous night shift work for one week, or floating night shifts $(14.1 \%$ - 214/1521), where a nurse is asked to occasionally do night shift work as needed. In addition, only $15.5 \%(236 / 1521)$ of the nurses indicated that they liked night shift work, while the remainder indicated that they disliked it $(49.1 \%-747 / 1521)$ or preferred it occasionally $(35.4 \%-538 / 121)$. Almost a quarter $(24.5 \%$ - 373/1521) had worked more than 20 weeks of night shifts in the year preceding the survey.
Table 1. Sociodemographic Characteristics of the Participants $(\mathrm{N}=1521)$

\begin{tabular}{|c|c|c|c|}
\hline Variable & Characteristics & Frequency & Percentage \\
\hline \multirow[t]{4}{*}{ Age group (years) } & $21-30$ & 838 & 55.1 \\
\hline & $31-40$ & 608 & 40.0 \\
\hline & $41-50$ & 65 & 4.3 \\
\hline & $>50$ & 10 & 0.7 \\
\hline \multirow[t]{2}{*}{ Gender } & Male & 788 & 51.8 \\
\hline & Female & 733 & 48.2 \\
\hline \multirow[t]{4}{*}{ Nationality } & Saudi & 1406 & 92.4 \\
\hline & Philippines & 60 & 3.9 \\
\hline & India & 36 & 2.4 \\
\hline & Others & 19 & 1.2 \\
\hline \multirow[t]{4}{*}{ Marital status } & Married & 1005 & 66.1 \\
\hline & Single & 466 & 30.6 \\
\hline & Divorced & 37 & 2.4 \\
\hline & Separated & 13 & 0.9 \\
\hline \multirow[t]{3}{*}{ Religion } & Muslim & 1443 & 94.9 \\
\hline & Christian & 64 & 4.2 \\
\hline & Others & 14 & 0.9 \\
\hline \multirow[t]{4}{*}{ Education } & Diploma & 888 & 58.4 \\
\hline & Bachelor's degree & 514 & 33.8 \\
\hline & $\begin{array}{l}\text { Postgraduate } \\
\text { diploma }\end{array}$ & 82 & 5.4 \\
\hline & $\begin{array}{c}\text { Master's } \\
\text { degree/PhD }\end{array}$ & 37 & 2.4 \\
\hline \multirow[t]{4}{*}{ Work experience (years) } & $1-3$ & 405 & 26.6 \\
\hline & $4-6$ & 402 & 26.4 \\
\hline & $7-10$ & 363 & 23.9 \\
\hline & $>10$ & 351 & 23.1 \\
\hline \multirow[t]{3}{*}{ Nursing position } & Staff nurse & 1117 & 73.4 \\
\hline & In-charge nurse & 194 & 12.8 \\
\hline & Nursing supervisor & 210 & 13.8 \\
\hline \multirow[t]{7}{*}{ Work department } & Emergency & 397 & 26.1 \\
\hline & Intensive care unit & 148 & 9.7 \\
\hline & Surgical & 130 & 8.5 \\
\hline & Medical & 138 & 9.1 \\
\hline & $\begin{array}{l}\text { Obstetrics \& } \\
\text { Gynecology }\end{array}$ & 113 & 7.4 \\
\hline & Pediatrics & 81 & 5.3 \\
\hline & Other & 514 & 33.8 \\
\hline \multirow[t]{13}{*}{ Region } & Makkah & 330 & 21.7 \\
\hline & Madina & 91 & 6.0 \\
\hline & Riyadh & 130 & 8.5 \\
\hline & Eastern region & 139 & 9.1 \\
\hline & Qassim & 75 & 4.9 \\
\hline & Hail & 23 & 1.5 \\
\hline & Aljouf & 73 & 4.8 \\
\hline & Tabuk & 120 & 7.9 \\
\hline & Northern borders & 126 & 8.3 \\
\hline & Asser & 258 & 17.0 \\
\hline & Baha & 6 & 0.4 \\
\hline & Jazan & 104 & 6.8 \\
\hline & Najran & 46 & 3.0 \\
\hline \multirow[t]{3}{*}{ Night shift pattern } & Rotating & 1142 & 75.1 \\
\hline & Fixed & 165 & 10.8 \\
\hline & Floating & 214 & 14.1 \\
\hline \multirow[t]{3}{*}{ Likes night shift work } & Yes & 236 & 15.5 \\
\hline & No & 747 & 49.1 \\
\hline & Sometimes & 538 & 35.4 \\
\hline \multirow[t]{5}{*}{$\begin{array}{l}\text { Night shifts done in the } \\
\text { past one year (weeks) }\end{array}$} & $1-5$ & 176 & 11.6 \\
\hline & $6-10$ & 164 & 10.8 \\
\hline & $11-20$ & 267 & 17.6 \\
\hline & $>20$ & 373 & 24.5 \\
\hline & I cannot remember & 541 & 35.6 \\
\hline
\end{tabular}

\subsection{Psychological and Social Effects of Night Shift Work}

The nurses' responses to the questions regarding the psychological and social effects of night shift work are as shown in Table 2. 
Table 2. Participants' Level of Agreement with the Psychological and Social Effects of Night Shift Work

\begin{tabular}{|c|c|c|c|c|c|}
\hline Variable & $\begin{array}{l}\text { Strongly } \\
\text { Agree }\end{array}$ & Agree & Disagree & $\begin{array}{l}\text { Strongly } \\
\text { Disagree }\end{array}$ & Mean score \\
\hline & $\mathrm{n}(\%)$ & $\mathrm{n}(\%)$ & $\mathrm{n}(\%)$ & $\mathrm{n}(\%)$ & $\pm \mathrm{SD}$ \\
\hline \multicolumn{6}{|l|}{ Psychological Effect } \\
\hline $\begin{array}{l}\text { I can maintain a positive attitude and behavior during night } \\
\text { shift work. }\end{array}$ & $113(7.4)$ & $762(50.1)$ & $418(27.5)$ & $228(15.0)$ & $2.5(0.8)$ \\
\hline I am satisfied with my night work schedule. & $44(2.9)$ & $447(29.4)$ & $581(38.2)$ & $449(29.5)$ & $2.9(0.8)$ \\
\hline I don't feel depressed and isolated when working night shifts. & $84(5.5)$ & $439(28.9)$ & $547(36.0)$ & $451(29.7)$ & $2.9(0.9)$ \\
\hline $\begin{array}{l}\text { I am mentally prepared so I can make the right decisions } \\
\text { during night shift work. }\end{array}$ & $104(6.8)$ & $695(45.7)$ & $481(31.6)$ & $241(15.8)$ & $2.6(0.8)$ \\
\hline $\begin{array}{l}\text { Night shift nurses get enough recognition and appreciation } \\
\text { compared to day shift nurses. }\end{array}$ & $53(3.5)$ & $224(14.7)$ & $502(33.0)$ & $742(48.8)$ & $3.3(0.8)$ \\
\hline \multicolumn{6}{|l|}{ Social Effect } \\
\hline $\begin{array}{l}\text { I am able to maintain social relationships when working night } \\
\text { shifts (e.g. family, friends). }\end{array}$ & $30(2.0)$ & $232(15.3)$ & $534(35.1)$ & $725(47.7)$ & $3.3(0.8)$ \\
\hline $\begin{array}{l}\text { I can manage my home and attend to my family needs when } \\
\text { working night shifts. }\end{array}$ & $33(2.2)$ & $276(18.1)$ & $559(36.8)$ & $653(42.9)$ & $3.2(0.8)$ \\
\hline My family understand my night schedule and supports me. & $83(5.5)$ & $568(37.3)$ & $492(32.3)$ & $378(24.9)$ & $2.8(0.9)$ \\
\hline $\begin{array}{l}\text { Home-Work transportation is handy (easy) when working } \\
\text { night shifts. }\end{array}$ & $100(6.6)$ & $504(33.1)$ & 446 (29.3) & $471(31.0)$ & $2.9(0.9)$ \\
\hline $\begin{array}{l}\text { I can manage my routine activities and social life (Prayers, } \\
\text { sports, shopping, festivals, etc.). }\end{array}$ & $49(3.2)$ & $266(17.5)$ & $565(37.1)$ & $641(42.1)$ & $3.2(0.8)$ \\
\hline
\end{tabular}

*Scores: strongly agree [1], agree [2], disagree [3], strongly disagree [4]; SD = Standard deviation.

The responses showed that their perception that: "Night shift nurses get enough recognition and appreciation compared to day shift nurses" (Mean 3.3; SD 0.8) ranked highest and their ability to maintain a positive attitude ranked lowest (Mean 2.5; SD 0.8) in their rating of the psychological effects of night shift work. Table 2 also reports on the social effects of night shift work. This showed that: "I am able to maintain social relationships when working night shifts (e.g. family, friends)" (Mean 3.3; SD 0.8) ranked highest and family support, which read thus: "My family understand my night schedule and support me" (Mean 2.8; SD 0.9) ranked lowest in their rating of the social effects of night shift work.

The median (mean) overall scores (minimum 5, maximum 20) of the psychological and social effects of night shift work according to the nurses' demographic profile are as shown in Table 3.

Table 3. Mean Overall Scores of the Psychosocial Effects of Night Shift Work According to the Participants' Sociodemographic Characteristics

\begin{tabular}{|c|c|c|c|c|c|c|}
\hline & \multicolumn{3}{|c|}{ Psychological impact } & \multicolumn{3}{|c|}{ Social impact } \\
\hline & Mean & Median (range) & P-value & Mean & Median (range) & P-value \\
\hline Age group (years) & & & $<0.001$ & & & $<0.001$ \\
\hline $21-30$ & 14.3 & $14.0(5,20)$ & & 15.5 & $15.0(5,20)$ & \\
\hline $31-40$ & 14.2 & $14.0(5,20)$ & & 15.3 & $15.0(5,20)$ & \\
\hline $41-50$ & 12.8 & $12.0(8,20)$ & & 14.0 & $14.0(5,20)$ & \\
\hline$>50$ & 11.1 & $11.0(5,20)$ & & 10.6 & $10.5(5,13)$ & \\
\hline Gender & & & 0.003 & & & $<0.001$ \\
\hline Male & 13.9 & $14.0(5,20)$ & & 14.8 & $15.0(5,20)$ & \\
\hline Female & 14.5 & $14.0(5,20)$ & & 15.8 & $16.0(5,20)$ & \\
\hline Nationality & & & $<0.001$ & & & $<0.001$ \\
\hline Saudi & 14.4 & $14.0(5,20)$ & & 15.5 & $15.0(5,20)$ & \\
\hline Philippines & 11.8 & $11.5(9,17)$ & & 12.5 & $12.0(9,20)$ & \\
\hline India & 11.4 & $11.0(7,16)$ & & 12.1 & $12.0(7,17)$ & \\
\hline Others & 12.5 & $11.0(9,20)$ & & 13.1 & $13.0(7,18)$ & \\
\hline Marital Status & & & 0.002 & & & 0.023 \\
\hline Married & 14.4 & $14.0(5,20)$ & & 15.4 & $15.0(5,20)$ & \\
\hline Single & 13.7 & $13.0(5,20)$ & & 15.0 & $15.0(5,20)$ & \\
\hline Divorced & 14.2 & $14.0(10,19)$ & & 15.2 & $15.0(10,20)$ & \\
\hline Separated & 13.9 & $14.0(5,18)$ & & 13.7 & $13.0(10,20)$ & \\
\hline Religion & & & $<0.001$ & & & $<0.001$ \\
\hline Muslim & 14.3 & $14.0(5,20)$ & & 15.5 & $15.0(5,20)$ & \\
\hline Christian & 11.4 & $11.0(7,20)$ & & 12.0 & $12.0(7,20)$ & \\
\hline Others & 12.3 & $12.0(9,16)$ & & 13.1 & $13.5(10,17)$ & \\
\hline
\end{tabular}




\begin{tabular}{|c|c|c|c|c|c|c|}
\hline & \multicolumn{3}{|c|}{ Psychological impact } & \multicolumn{3}{|c|}{ Social impact } \\
\hline & Mean & Median (range) & P-value & Mean & Median (range) & P-value \\
\hline Education & & & $<0.001$ & & & $<0.001$ \\
\hline Diploma & 14.5 & $14.0(5,20)$ & & 15.6 & $16.0(5,20)$ & \\
\hline Bachelor's degree & 13.7 & $13.0(5,20)$ & & 14.9 & $15.0(5,20)$ & \\
\hline Postgraduate diploma & 14.0 & $14.0(6,20)$ & & 15.2 & $15.0(5,20)$ & \\
\hline Master's degree/PhD & 13.2 & $13.0(5,20)$ & & 14.1 & $14.0(5,20)$ & \\
\hline Work experience (years) & & & $<0.001$ & & & 0.019 \\
\hline $1-3$ & 13.7 & $13.0(5,20)$ & & 15.0 & $15.0(5,20)$ & \\
\hline $4-6$ & 14.6 & $14.0(5,20)$ & & 15.6 & $16.0(5,20)$ & \\
\hline $7-10$ & 14.4 & $14.0(5,20)$ & & 15.5 & $16.0(6,20)$ & \\
\hline$>10$ & 14.0 & $14.0(5,20)$ & & 15.0 & $15.0(5,20)$ & \\
\hline Nursing position & & & 0.018 & & & 0.026 \\
\hline Staff nurse & 14.3 & $14.0(5,20)$ & & 15.4 & $15.0(5,20)$ & \\
\hline In-charge nurse & 13.9 & $13.5(5,20)$ & & 15.1 & $15.0(5,20)$ & \\
\hline Nursing supervisor & 13.7 & $14.0(5,20)$ & & 14.7 & $15.0(5,20)$ & \\
\hline Department & & & $<0.001$ & & & $<0.001$ \\
\hline Emergency & 13.6 & $13.0(6,20)$ & & 14.7 & $15.0(5,20)$ & \\
\hline Intensive care unit & 13.9 & $14.0(6,20)$ & & 15.4 & $15.0(5,20)$ & \\
\hline Surgical & 14.5 & $14.0(5,20)$ & & 15.7 & $16.0(5,20)$ & \\
\hline Medical & 14.7 & $15.0(7,20)$ & & 15.5 & $15.0(5,20)$ & \\
\hline Obstetrics \& Gynecology & 15.0 & $15.0(5,20)$ & & 16.1 & $17.0(5,20)$ & \\
\hline Pediatrics & 15.0 & $15.0(9,20)$ & & 16.4 & $17.0(6,20)$ & \\
\hline Other & 14.2 & $14.0(5,20)$ & & 15.2 & $15.0(5,20)$ & \\
\hline Region & & & $<0.001$ & & & $<0.001$ \\
\hline Makkah & 13.9 & $13.0(5,20)$ & & 15.1 & $15.0(5,20)$ & \\
\hline Madina & 14.0 & $13.0(6,20)$ & & 14.9 & $15.0(5,20)$ & \\
\hline Riyadh & 13.7 & $14.0(5,20)$ & & 14.9 & $15.0(5,20)$ & \\
\hline Eastern region & 14.8 & $14.0(6,20)$ & & 15.7 & $16.0(6,20)$ & \\
\hline Qassim & 13.8 & $13.0(5,20)$ & & 15.2 & $15.0(5,20)$ & \\
\hline Hail & 15.6 & $16.0(11,20)$ & & 16.7 & $17.0(10,20)$ & \\
\hline Aljouf & 14.1 & $14.0(5,20)$ & & 15.2 & $15.0(7,20)$ & \\
\hline Tabuk & 15.1 & $15.0(5,20)$ & & 15.9 & $16.0(5,20)$ & \\
\hline Northern borders & 14.7 & $15.0(5,20)$ & & 15.8 & $16.0(5,20)$ & \\
\hline Asser & 14.3 & $14.0(7,20)$ & & 15.5 & $16.0(6,20)$ & \\
\hline Baha & 15.2 & $15.0(13,18)$ & & 17.0 & $16.5(15,20)$ & \\
\hline Jazan & 14.1 & $14.0(7,20)$ & & 15.3 & $15.0(5,20)$ & \\
\hline Najran & 11.4 & $11.0(7,16)$ & & 12.3 & $12.0(9,19)$ & \\
\hline Night shift pattern & & & $<0.001$ & & & 0.014 \\
\hline Rotating & 14.4 & $14.0(5,20)$ & & 15.4 & $15.0(5,20)$ & \\
\hline Fixed & 13.7 & $13.0(5,20)$ & & 14.6 & $14.0(5,20)$ & \\
\hline Floating & 13.5 & $13.5(5,20)$ & & 14.9 & $15.0(5,20)$ & \\
\hline Likes night shift work & & & $<0.001$ & & & $<0.001$ \\
\hline Yes & 11.5 & $11.0(5,20)$ & & 12.4 & $12.0(5,20)$ & \\
\hline No & 15.7 & $16.0(6,20)$ & & 16.7 & $17.0(5,20)$ & \\
\hline Sometimes & 13.2 & $13.0(5,20)$ & & 14.6 & $14.0(5,20)$ & \\
\hline $\begin{array}{l}\text { Night shifts done in the past one } \\
\text { year (weeks) }\end{array}$ & & & $<0.001$ & & & $<0.001$ \\
\hline $1-5$ & 13.1 & $13.0(5,20)$ & & 14.4 & $14.0(5,20)$ & \\
\hline $6-10$ & 13.5 & $13.0(6,20)$ & & 14.6 & $15.0(6,20)$ & \\
\hline $11-20$ & 14.3 & $14.0(5,20)$ & & 15.3 & $15.0(5,20)$ & \\
\hline$>20$ & 15.7 & $15.0(6,20)$ & & 14.8 & $16.0(5,20)$ & \\
\hline I cannot remember & 14.2 & $14.0(5,20)$ & & 15.5 & $15.0(5,20)$ & \\
\hline
\end{tabular}

The overall median psychological effect score was 14.0 (maximum 20). The median scores for the psychological effects of night shift work were higher in younger nurses compared to older ones (14.0 vs. 11.0 ; $\mathrm{P}<0.001)$, higher in females $(\mathrm{P}=0.003)$, higher in Saudi nationals compared to non-Saudi nurses (median 14.0 vs. 11.0; $\mathrm{P}<0.001)$ and higher among nurses who were married compared to those who were single (median 14,0 vs. 13.0; $\mathrm{P}=0.002)$. Furthermore, the median scores for the psychological effects of night shift work were higher 
among Muslim nurses $(\mathrm{P}<0.001)$, nurses with a diploma qualification ( $\mathrm{P}<0.001)$, with more years of work experience $(\mathrm{P}<0.001)$ and staff nurses $(\mathrm{P}=0.018)$. In addition, the median scores for the psychological effects of night shift work were higher among nurses in the medical, surgical and obstetrics and gynecology departments compared with other departments (all median 15.0; $\mathrm{P}<0.001$ ) and higher among nurses in the Hail region compared with other regions $(\mathrm{P}<0.001)$. Furthermore, the median scores for the psychological effects of night shift work were higher among nurses involved in rotating night shift work $(\mathrm{P}<0.001)$, among those who disliked night shift work ( $\mathrm{P}<0.001)$ and among nurses who had had over 20 weeks of night shift work in the preceding year.

The overall median social effect score was 15.0 (maximum 20). Similarly, the median scores for the social effects of night shift work were higher in younger nurses compared to older ones (15.0 vs. 10.5; $\mathrm{P}<0.001)$, higher in females ( 16.0 vs. 15.0; $\mathrm{P}<0.001)$, higher in Saudi nurses compared to non-Saudi nurses (median 15.0 vs. 12.0; $\mathrm{P}<0.001)$ and higher among nurses who were married compared to those who were single or separated (median;
$\mathrm{P}=0.023)$. Furthermore, the median scores for the social effects of night shift work were higher among Muslim nurses $(\mathrm{P}<0.001)$, nurses with a diploma qualification $(\mathrm{P}<0.001)$, with more years of work experience $(\mathrm{P}=0.019)$ and staff nurses $(P=0.026)$. In addition, the median scores for the social effects of night shift work were higher among nurses in the medical and obstetrics and gynecology departments compared with other departments (all median 17.0; $\mathrm{P}<0.001$ ) and higher among nurses in the Baha region compared with other regions $(\mathrm{P}<0.001)$. Furthermore, the median scores for the social effects of night shift work were higher among nurses involved in rotating night shift work $(\mathrm{P}=0.014)$, among those who disliked night shift work $(\mathrm{P}<0.001)$ and among nurses who had had over 20 weeks of night shift work in the preceding year.

\subsection{Psychological and Social Impact of Night Shift Work and associated factors}

Table 4 summarizes the proportion of the participants who reported a psychological impact of night shift work and its associated factors.

Table 4. Multivariable Logistic Regression Analysis of Factors Associated with Significant Psychological Impact of Night Shift Work

\begin{tabular}{|c|c|c|c|c|c|}
\hline \multirow[t]{2}{*}{ Variable } & \multicolumn{2}{|c|}{ Psychological impact } & \multirow{2}{*}{$\begin{array}{c}\text { Crude OR } \\
(95 \% \text { C. I. })\end{array}$} & \multirow{2}{*}{$\begin{array}{c}\text { Adjusted OR } \\
(95 \% \text { C. I. })\end{array}$} & \multirow{2}{*}{$\begin{array}{l}\text { Adjusted } \\
\text { P-value }\end{array}$} \\
\hline & No, n (\%) & Yes, n (\%) & & & \\
\hline \multicolumn{6}{|l|}{ Age group (years) } \\
\hline $21-30$ & $99(11.8)$ & $739(88.2)$ & $1.9(0.4-8.9)$ & $0.8(0.1-5.2)$ & 0.81 \\
\hline $31-40$ & $68(11.2)$ & $540(88.8)$ & $2.0(0.4-9.5)$ & $0.8(1.3-4.8)$ & 0.79 \\
\hline $41-50$ & $11(16.9)$ & $54(83.1)$ & $1.2(0.2-6.6)$ & $0.6(0.9-3.4)$ & 0.59 \\
\hline$>50$ & $2(20.0)$ & $8(80.0)$ & 1 & 1 & \\
\hline \multicolumn{6}{|l|}{ Gender } \\
\hline Male & $105(13.3)$ & $683(86.7)$ & 1 & 1 & \\
\hline Female & $75(10.2)$ & $658(89.8)$ & $1.3(1.0-1.8)$ & $1.6(1.1-2.6)$ & 0.032 \\
\hline \multicolumn{6}{|l|}{ Nationality } \\
\hline Saudi & $151(10.7)$ & $1255(89.3)$ & $3.8(1.4-10.2)$ & $3.5(1.1-10.9)$ & 0.033 \\
\hline Philippines & $11(18.3)$ & $49(81.7)$ & $2.1(0.6-6.6)$ & $7.3(1.4-36.5)$ & 0.016 \\
\hline India & $12(33.3)$ & $24(66.7)$ & $0.9(0.3-3.0)$ & $4.6(0.924 .6)$ & 0.076 \\
\hline Others & $6(31.6)$ & $13(68.4)$ & 1 & 1 & \\
\hline \multicolumn{6}{|l|}{ Marital Status } \\
\hline Married & $110(10.9)$ & $895(89.1)$ & 1 & 1 & \\
\hline Single & $65(13.9)$ & $401(86.1)$ & $0.8(0.5-1.1)$ & $0.9(0.6-1.4)$ & 0.63 \\
\hline Divorced & $4(10.8)$ & $33(89.2)$ & $1.0(0.4-2.9)$ & $1.1(0.4-3.6)$ & 0.85 \\
\hline Separated & $1(7.7)$ & $12(92.3)$ & $1.5(0.2-11.5)$ & $1.3(0.2-11.6)$ & 0.79 \\
\hline \multicolumn{6}{|l|}{ Religion } \\
\hline Muslim & $158(10.9)$ & $1285(89.1)$ & $3.3(1.1-10.5)$ & $2.9(0.5-18.2)$ & 0.25 \\
\hline Christian & $18(28.1)$ & $46(71.9)$ & $1.0(0.3-3.7)$ & $0.6(0.1-2.6)$ & 0.48 \\
\hline Others & $4(28.6)$ & $10(71.4)$ & 1 & & \\
\hline \multicolumn{6}{|l|}{ Education } \\
\hline Diploma & $90(10.1)$ & $798(89.9)$ & $2.1(0.9-4.8)$ & $1.4(0.5-3.6)$ & 0.52 \\
\hline Bachelor's degree & $73(14.2)$ & $441(85.8)$ & $1.4(0.6-3.3)$ & $1.2(0.5-3.2)$ & 0.69 \\
\hline Postgraduate diploma & $10(12.2)$ & $72(87.8)$ & $1.7(0.6-4.8)$ & $1.1(0.3-3.4)$ & 0.95 \\
\hline Master's degree/PhD & $7(18.9)$ & $30(81.1)$ & 1 & 1 & \\
\hline \multicolumn{6}{|c|}{ Work experience (years) } \\
\hline $1-3$ & $62(15.3)$ & $343(84.7)$ & 1 & 1 & \\
\hline $4-6$ & $38(9.5)$ & $364(90.5)$ & $1.7(1.1-2.7)$ & $1.5(0.9-2.4)$ & 0.11 \\
\hline $7-10$ & $38(10.5)$ & $325(89.5)$ & $1.5(1.1-2.4)$ & $1.5(0.9-2.6)$ & 0.16 \\
\hline$>10$ & $42(12.0)$ & $309(88.0)$ & $1.3(0.9-2.0)$ & $1.4(0.7-2.8)$ & 0.32 \\
\hline \multicolumn{6}{|l|}{ Nursing position } \\
\hline Staff nurse & $130(11.6)$ & $987(88.4)$ & $1.3(0.8-1.9)$ & $1.1(0.7-2.0)$ & 0.64 \\
\hline In-charge nurse & $20(10.3)$ & $174(89.7)$ & $1.5(0.8-2.7)$ & $1.4(0.7-2.7)$ & 0.36 \\
\hline Nursing supervisor & $30(14.3)$ & $180(85.7)$ & 1 & 1 & \\
\hline
\end{tabular}




\begin{tabular}{|c|c|c|c|c|c|}
\hline \multirow[t]{2}{*}{ Variable } & \multicolumn{2}{|c|}{ Psychological impact } & \multirow{2}{*}{$\begin{array}{l}\text { Crude OR } \\
\text { (95\% C. I.) }\end{array}$} & \multirow{2}{*}{\begin{tabular}{|c|} 
Adjusted OR \\
$(95 \%$ C. I. $)$
\end{tabular}} & \multirow{2}{*}{$\begin{array}{c}\text { Adjusted } \\
\text { P-value }\end{array}$} \\
\hline & No, n (\%) & Yes, n (\%) & & & \\
\hline \multicolumn{6}{|l|}{ Department } \\
\hline Emergency & $58(14.6)$ & $339(85.4)$ & 1 & 1 & \\
\hline Intensive care unit & $15(10.1)$ & $133(89.9)$ & $1.5(0.8-2.8)$ & $1.2(0.6-2.4)$ & 0.55 \\
\hline Surgical & $9(6.9)$ & $121(93.1)$ & $2.3(1.1-4.8)$ & $1.6(0.8-3.6)$ & 0.21 \\
\hline Medical & $11(8.0)$ & $127(92.0)$ & $2.0(1.1-3.9)$ & $1.5(0.7-3.2)$ & 0.25 \\
\hline Obstetrics \& Gynecology & $13(11.5)$ & $100(88.5)$ & $1.3(0.7-2.5)$ & $0.7(0.3-1.5)$ & 0.40 \\
\hline Pediatrics & $8(9.9)$ & $73(90.1)$ & $1.6(0.7-3.4)$ & $0.9(0.4-2.1)$ & 0.76 \\
\hline Other & $66(12.8)$ & $448(87.2)$ & $1.2(0.8-1.7)$ & $1.1(0.7-1.7)$ & 0.67 \\
\hline \multicolumn{6}{|l|}{ Night shift pattern } \\
\hline Rotating & $119(10.4)$ & $1023(89.6)$ & $1.9(1.3-2.8)$ & $2.1(1.3-3.5)$ & 0.002 \\
\hline Fixed & $22(13.3)$ & $143(86.7)$ & $1.5(0.8-2.6)$ & $2.2(1.1-4.3)$ & 0.018 \\
\hline Floating & $39(18.2)$ & $175(81.8)$ & 1 & 1 & \\
\hline \multicolumn{6}{|l|}{ Likes night shift work } \\
\hline Yes & $77(32.6)$ & $159(67.4)$ & 1 & 1 & \\
\hline No & $31(4.1)$ & $716(95.9)$ & $11.1(7.1-17.6)$ & $11.5(7.1-18.8)$ & $<0.001$ \\
\hline Sometimes & $72(13.4)$ & $466(86.6)$ & $3.1(2.2-4.5)$ & $3.1(2.1-4.7)$ & $<0.001$ \\
\hline \multicolumn{6}{|c|}{ Night shifts done in the past one year (weeks) } \\
\hline $1-5$ & $35(19.9)$ & $141(80.1)$ & 1 & 1 & \\
\hline $6-10$ & $18(11.0)$ & $146(89.0)$ & $2.0(1.1-3.7)$ & $2.1(1.1-4.1)$ & 0.043 \\
\hline $11-20$ & $30(11.2)$ & $237(88.8)$ & $2.0(1.1-3.3)$ & $1.6(0.9-3.0)$ & 0.13 \\
\hline$>20$ & $35(9.4)$ & $338(90.6)$ & $2.4(1.4-4.0)$ & $2.4(1.3-4.3)$ & 0.004 \\
\hline I cannot remember & $62(11.5)$ & $479(88.5)$ & $1.9(1.2-3.0)$ & $1.6(0.9-2.7)$ & 0.08 \\
\hline
\end{tabular}

$* \mathrm{OR}=$ odds ratio $; 95 \%$ C.I. $=95 \%$ confidence interval.

A total of $88.2 \%(1341 / 1521)$ of the participants reported a psychological impact due to night shift work. After adjusting for confounders, being a female nurse (adjusted odds ratio [aOR] 1.6; 95\% CI 1.1 - 2.6), being a Saudi (aOR 3.5; 95\% CI 1.1 - 10.9) or Filipino national (aOR 7.3; 95\% CI 1.4 - 36.5) and being involved in rotating (aOR 2.1; 95\% CI $1.3-3.5$ ) or fixed (aOR 2.2; 95\% CI 1.1 - 4.3) night shift work were significant predictors of having a psychological impact due to night shift work (Table 4). Furthermore, dislike
(aOR 11.5; 95\% CI $7.1-18.8$ ) or occasional dislike for night shift work (aOR 3.1; 95\% CI 2.1 - 4.7) and having had 6-10 weeks (aOR 2.1; 95\% CI $1.1-4.1$ ) or over 20 weeks of night shift work in the preceding year (aOR 2.4; $95 \%$ CI $1.3-4.3$ ) were other independent predictors of having a psychological impact due to night shift work (Table 4).

Table 5 summarizes the proportion of the participants who reported a social impact of night shift work and its associated factors.

Table 5. Multivariable Logistic Regression Analysis of Factors Associated with Significant Social Impacts of Night Shift Work

\begin{tabular}{|c|c|c|c|c|c|}
\hline \multirow[t]{2}{*}{ Variable } & \multicolumn{2}{|c|}{ Social impact } & \multirow{2}{*}{$\begin{array}{l}\text { Crude OR } \\
\text { (95\% C. I.) } \\
\end{array}$} & \multirow{2}{*}{$\begin{array}{c}\text { Adjusted OR } \\
\text { (95\% C. I.) } \\
\end{array}$} & \multirow{2}{*}{$\begin{array}{c}\text { Adjusted } \\
\text { P-value } \\
\end{array}$} \\
\hline & No, n (\%) & Yes, n (\%) & & & \\
\hline \multicolumn{6}{|c|}{ Age group (years) } \\
\hline $21-30$ & $68(8.1)$ & $770(91.9)$ & $11.3(3.2-40.1)$ & $5.1(0.9-30.2)$ & 0.08 \\
\hline $31-40$ & $52(8.6)$ & $556(91.4)$ & $10.7(3.0-38.1)$ & $3.8(0.7-20.5)$ & 0.13 \\
\hline $41-50$ & $13(20.0)$ & $52(80.0)$ & $4.0(1.1-15.9)$ & $1.4(0.2-8.1)$ & 0.73 \\
\hline$>50$ & $5(50)$ & $5(50)$ & 1 & $1.3(0.8-2.2)$ & 0.36 \\
\hline \multicolumn{6}{|l|}{ Gender } \\
\hline Male & $76(9.6)$ & $712(90.4)$ & 1 & 1 & \\
\hline Female & $62(8.5)$ & $671(91.5)$ & $1.2(0.8-1.6)$ & $1.3(0.8-2.2)$ & 0.36 \\
\hline \multicolumn{6}{|l|}{ Nationality } \\
\hline Saudi & $110(7.8)$ & $1296(92.2)$ & $1.4(0.3-6.1)$ & $1.1(0.2-5.6)$ & 0.95 \\
\hline Philippines & $16(26.7)$ & $44(73.3)$ & $0.3(0.1-1.6)$ & $1.1(0.2-7.3)$ & 0.93 \\
\hline India & $10(27.8)$ & $26(72.2)$ & $0.3(0.1-1.6)$ & $1.3(0.2-9.9)$ & 0.79 \\
\hline Others & $2(10.5)$ & $17(89.5)$ & 1 & 1 & \\
\hline \multicolumn{6}{|c|}{ Marital Status } \\
\hline Married & $90(9.0)$ & $915(91.0)$ & $1.8(0.4-8.5)$ & $2.3(0.4-12.1)$ & 0.33 \\
\hline Single & $42(9.0)$ & $424(91.0)$ & $1.8(0.4-8.6)$ & $2.8(0.5-15.4)$ & 0.23 \\
\hline Divorced & $4(10.8)$ & $33(89.2)$ & $1.5(0.2-9.3)$ & $2.3(0.3-17.2)$ & 0.43 \\
\hline Separated & $2(15.4)$ & $11(84.6)$ & 1 & 1 & \\
\hline \multicolumn{6}{|l|}{ Religion } \\
\hline Muslim & $115(8.0)$ & $1328(92.0)$ & $3.1(0.9-11.5)$ & $2.9(0.5-18.9)$ & 0.26 \\
\hline Christian & $20(31.2)$ & $44(68.8)$ & $0.6(0.2-2.4)$ & $0.6(0.1-3.1)$ & 0.56 \\
\hline Others & $3(21.4)$ & $11(78.8)$ & 1 & 1 & \\
\hline
\end{tabular}




\begin{tabular}{|c|c|c|c|c|c|}
\hline \multirow[t]{2}{*}{ Variable } & \multicolumn{2}{|c|}{ Social impact } & \multirow{2}{*}{$\begin{array}{l}\text { Crude OR } \\
(95 \% \text { C. I.) }\end{array}$} & \multirow{2}{*}{$\begin{array}{c}\text { Adjusted OR } \\
(95 \% \text { C. I.) }\end{array}$} & \multirow{2}{*}{$\begin{array}{c}\text { Adjusted } \\
\text { P-value }\end{array}$} \\
\hline & No, $n(\%)$ & Yes, n (\%) & & & \\
\hline \multicolumn{6}{|l|}{ Education } \\
\hline Diploma & $70(7.9)$ & $818(92.1)$ & $2.3(0.9-5.6)$ & $1.3(0.5-3.9)$ & 0.57 \\
\hline Bachelor's degree & $55(10.7)$ & $459(89.3)$ & $1.6(0.6-4.0)$ & $1.3(0.5-3.8)$ & 0.62 \\
\hline Postgraduate diploma & $7(8.5)$ & $75(91.5)$ & $2.1(0.6-6.7)$ & $1.1(0.3-4.1)$ & 0.86 \\
\hline Master's degree/PhD & $6(16.2)$ & $31(83.8)$ & 1 & 1 & \\
\hline \multicolumn{6}{|l|}{ Work experience (years) } \\
\hline $1-3$ & $42(10.4)$ & $363(89.6)$ & 1 & 1 & \\
\hline $4-6$ & $33(8.2)$ & $369(91.8)$ & $1.3(0.8-2.1)$ & $1.2(0.7-2.0)$ & 0.58 \\
\hline $7-10$ & $29(8.0)$ & $334(92.0)$ & $1.3(0.8-2.2)$ & $1.6(0.9-3.0)$ & 0.14 \\
\hline$>10$ & $34(9.7)$ & $317(90.3)$ & $1.1(0.7-1.7)$ & $2.1(1.0-4.6)$ & 0.06 \\
\hline \multicolumn{6}{|l|}{ Nursing position } \\
\hline Staff nurse & $94(8.4)$ & 1023 (91.6) & $1.5(0.9-2.4)$ & $1.3(0.7-2.4)$ & 0.37 \\
\hline In-charge nurse & $19(9.8)$ & $175(90.2)$ & $1.2(0.7-2.3)$ & $1.3(0.6-2.7)$ & 0.52 \\
\hline Nursing supervisor & $25(11.9)$ & $185(88.1)$ & 1 & & \\
\hline \multicolumn{6}{|l|}{ Department } \\
\hline Emergency & $43(10.8)$ & $354(89.2)$ & 1 & 1 & \\
\hline Intensive care unit & $10(6.8)$ & $138(93.2)$ & $1.7(0.8-3.4)$ & $1.2(0.5-2.6)$ & 0.67 \\
\hline Surgical & $10(7.7)$ & $120(92.3)$ & $1.5(0.7-3.0)$ & $1.0(0.4-2.2)$ & 0.99 \\
\hline Medical & $8(5.8)$ & $130(94.2)$ & $2.0(0.9-4.3)$ & $1.4(0.6-3.4)$ & 0.40 \\
\hline Obstetrics \& Gynecology & $12(10.6)$ & $101(89.4)$ & $1.1(0.5-2.0)$ & $0.6(0.3-1.3)$ & 0.20 \\
\hline Pediatrics & $6(7.4)$ & $75(92.6)$ & $1.5(0.6-3.7)$ & $0.8(0.3-2.3)$ & 0.74 \\
\hline Other & $49(9.5)$ & $465(90.5)$ & $1.2(0.8-1.8)$ & $1.2(0.7-1.9)$ & 0.54 \\
\hline \multicolumn{6}{|l|}{ Night shift pattern } \\
\hline Rotating & $89(7.8)$ & $1053(92.2)$ & $1.9(1.2-2.9)$ & $2.1(1.2-3.7)$ & 0.009 \\
\hline Fixed & 20() 12.1 & $145(87.9)$ & $1.1(0.6-2.1)$ & $1.8(0.9-3.8)$ & 0.09 \\
\hline Floating & $29(13.6)$ & $185(86.4)$ & 1 & 1 & \\
\hline \multicolumn{6}{|l|}{ Likes night shift work } \\
\hline Yes & $69(29.2)$ & $167(70.8)$ & 1 & 1 & \\
\hline No & $23(3.1)$ & $724(96.9)$ & $13.0(9.9-21.5)$ & $13.7(7.9-23.8)$ & $<0.001$ \\
\hline Sometimes & $46(8.6)$ & $492(91.4)$ & $4.4(2.9-6.7)$ & $4.8(3.0-7.6)$ & $<0.001$ \\
\hline \multicolumn{6}{|c|}{$\begin{array}{l}\text { Night shifts done in the past one year } \\
\text { (weeks) }\end{array}$} \\
\hline $1-5$ & $28(15.9)$ & $148(84.1)$ & 1 & 1 & \\
\hline $6-10$ & $16(9.8)$ & $148(90.2)$ & $1.8(0.9-3.4)$ & $1.5(0.7-3.2)$ & 0.30 \\
\hline $11-20$ & $22(8.2)$ & $245(91.8)$ & $2.1(1.2-3.8)$ & $1.8(0.9-3.6)$ & 0.12 \\
\hline$>20$ & $27(7.2)$ & $346(92.8)$ & $2.4(1.4-4.3)$ & $2.5(1.3-4.9)$ & 0.007 \\
\hline I cannot remember & $45(8.3)$ & $496(91.7)$ & $2.1(1.3-3.5)$ & $1.7(0.9-3.0)$ & 0.08 \\
\hline
\end{tabular}

$* \mathrm{OR}=$ odds ratio $; 95 \%$ C.I. $=95 \%$ confidence interval.

A total of $90.9 \%(1383 / 1521)$ of the participants reported a social impact due to night shift work. After adjusting for potential confounders, being involved in rotating (aOR 2.1; 95\% CI 1.2 - 3.7) night shift work, dislike for night shift work (aOR 13.7; 95\% CI $7.9-23.8$ ) or occasional dislike for night shift work (aOR 4.8; 95\% CI 3.0 - 7.6) and having had over 20 weeks of night shift work in the preceding year (aOR 2.5; 95\% CI $1.3-4.9$ ) were independent predictors of having a social impact due to night shift work (Table 5).

\section{Discussion}

This large survey has indicated that the concern that night shift nurses do not get enough recognition and appreciation compared to day shift nurses for the work they do drives the psychological effects of the shift work among nurses in SA and that, despite this concern, they retain their ability to maintain a positive attitude to the work. In addition, the survey has shown that over four-fifths of nurses dislike or occasionally dislike night shift work. Thus, it is likely that these concerns, coupled with their dislike for night shift work, drives the psychological impact of night shift work among nurses and predisposes them to develop mental disorders, such as anxiety $[14,15]$, depression and mood disorders, coupled with shift work's other physiological effects $[14,15,17]$. This study's findings suggest that the development of strategies that reward night shift work may help lower the psychological effects on the nurses who are involved in the shift work. A systematic review by Li et al. suggested that the integration of napping during a night shift is beneficial to nurses' health, their psychomotor vigilance and their performance [33].

Furthermore, this study has shown that the social effects of night shift work are driven by the nurses' concerns about their inability to maintain social relationships while working night shifts and that this effect is lowered if they receive understanding and support from their family members. This finding is consistent with other studies that have shown that the nurses engaged in 
night shifts experience social isolation and lower partnership and job satisfaction rates [23,24,25,26]. Another study showed that lack of support from family members also increases the social effects of night shift work among nurses [21]. However, this study's findings have suggested that the social effects of night shift work could be lowered through the support and understanding of their work schedules from their family members.

This study has found that the mean and median scores of the psychological and social effects items were high, indicating the questionnaire's ease of use and the likelihood that a high proportion of the respondents experienced psychosocial issues due to the night shift work, although these effects varied across the participants' demographic profile, which is consistent with studies carried out elsewhere [22,31,32]. The study's findings have shown that almost $88 \%$ of the nurses engaged in night shift work had felt a psychological impact due to night shift work. Female nurses were almost twice as likely to experience the psychological effects of night shift work compared to male nurses, which might be due to additional responsibilities in their homes that prevent them from having adequate sleep or rest during the day. The reason why Saudi and Filipino nurses had a higher risk of experiencing the psychological impacts of night shift work is not clear and calls for further investigation. In addition, this study found that nurses involved in rotating or fixed night shift work were at least twice as likely to have a psychological impact due to night shift work. Moreover, having a dislike for night shift work and having over 20 weeks of night shift work in the preceding year were other risk factors that should be considered during the preparation of duty schedules for nurses in SA.

In addition, this study has found that almost $91 \%$ of the participants experienced a social impact due to night shift work. This indicates that there is a need for strategies to address the psychosocial impact of night shift work in the setting, probably through the creation of incentives, the introduction of support groups and support from the nurses' family members. Furthermore, being involved in rotating night shift work, dislike for night shift work and having had over 20 weeks of night shift work in the preceding year were factors that independently predicted the occurrence of social impacts due to night shift work among the nurses. The reasons for the high social impact of night shift work among the nurses are most likely intertwined with cultural and religious beliefs. A survey by Nasrabadi et al. in Iran reported that most of the respondents stated that working night shifts was associated with a negative socio-cultural image of themselves, as well as their family members and the general public [26]. Thus, these socio-cultural factors need to be explored further and should be considered during the preparation of work schedules for nurses working in the public sector in SA.

This study's strength is that it was carried out among a large population of nurses from all regions in Saudi Arabi and might, therefore, be representative of the situation in the country. Second, the study utilized a simple questionnaire that was easy to administer in various formats and, third, the study identified the independent predictors of experiencing psychosocial impacts due to night shift work. However, the study has some limitations.
First, this was a cross-sectional study and, therefore, the findings should not be used to make any causal inferences. Second, no formal sample size calculation was performed as it was planned that the survey would reach all nurses working in the public sector in all regions of the country. Third, the spread of public facilities in the regions might not be uniform, which might have led to differences in the proportion of nurses surveyed across the regions. Fourth, the study did not include an additional qualitative survey in order to triangulate the study findings. An additional qualitative study might have identified other socio-cultural themes driving the psychosocial impact of night shift work that this survey missed.

\section{Conclusion}

There is high psychosocial impact of night shift work among nurses in SA. This calls for further investigation to understand other contextual issues that might be contributing to the psychosocial impact of night shift work among nurses. In addition, there is a need to identify and develop ways to support nurses engaged in night shift work. Individuals who belong to the at-risk groups should be prioritized during preparation of duty schedules and their family members need to be encouraged to provide them with support.

\section{Acknowledgments}

The author would like to thank all the nurses who agreed to take part in this study for their valuable time and feedback.

\section{Disclosure}

The author reports no conflicts of interest in this work.

\section{References}

[1] Pati, A.K., Chandrawanshi, A. and Reinberg, A. "Shift work: consequences and management". Curr Sci. 8: 32-52. 2001.

[2] Books, C., Coody, L.C., Kauffman, R. and Abraham, S. "Night shift work and its health effects on nurses". Health Care Manag (Frederick). 36: 347-353. 2017.

[3] Dall'Ora, C., Griffiths, P., Ball, J., Simon, M. and Aiken, L.H. "Association of $12 \mathrm{~h}$ shifts and nurses' job satisfaction, burnout and intention to leave: findings from a cross-sectional study of 12 European countries". BMJ Open. 5: e008331. 2015.

[4] Kerkhof, G.A. "Shift work and sleep disorder comorbidity tend to go hand in hand". Chronobiol Int. 35: 1-10. 2017

[5] Cheng, P. and Drake, C.L. "Psychological impact of shift work". Curr Sleep Med Rep. 4: 104-109. 2018.

[6] Khamisa, N., Peltzer, K. and Oldenburg, B. "Burnout in relation to specific contributing factors and health outcomes among nurses: a systematic review". Int J Environ Res Public Health. 10: 2214-2240. 2013.

[7] Almajwal, A.M. "Stress, shift duty, and eating behavior among nurses in Central Saudi Arabia". Saudi Med J. 37: 191-198. 2016.

[8] Vidotti, V., Ribeiro, R.P., Galdino, M.J.Q. and Martins, J.T. "Burnout Syndrome and shift work among the nursing staff". Rev Lat Am Enfermagem. 26: e3022. 2018.

[9] Selivi, Y., Özdemir, P.G., Özdemir, O., Aydin, A. and Beşiroğlu, L. "Influence of night shift work on psychologic state and quality 
of life in health workers". Düşünen Adam: Journal of Psychiatry and Neurological Sciences 23: 238-243. 2010.

[10] Proper, K.I., van de Langenberg, D., Rodenburg, W., Vermeulen, R.C.H., van der Beek, A.J. and van Steeg, H. "The relationship between shift work and metabolic risk factors: a systematic review of longitudinal studies". Am J Prev Med. 5: e147-e157. 2016.

[11] Frost, P., Kolstad, H.A. and Bonde, J.P. "Shift work and the risk of ischemic heart disease - a systematic review of the epidemiologic evidence". Scand J Work Environ Health. 35: 163-179. 2009

[12] Bonde, J.P., Hansen, J., Kolstad, H.A., Mikkelsen, S., Olsen, J.H., and Blask, D.E. "Work at night and breast cancer--report on evidence-based options for preventive actions". Scand J Work Environ Health. 38: 380-90. 2012.

[13] Zaki, N.W.F., Denewar, K.A.F., El Sherif, M.A.F., Elweheidy, A., Ibrahim, H.F. and Pandi-Perumal, S.R. "Psychological correlates of shift-work sleep disorder among a sample of Egyptian nurses". Arab J Psychiatr. 27: 127-143. 2016.

[14] Angerer, P., Schmook, R., Elfantel, I. and Li, J. "Night work and the risk of depression". Dtsch Arztebl Int. 114: 404-411. 2017

[15] Cheung, T. and Yip, P.S. "Depression, anxiety and symptoms of stress among Hong Kong nurses: a cross-sectional study". Int $J$ Environ Res Public Health. 12: 11072-11100. 2015.

[16] Thompson, B.J., Stock, M.S. and Banuelas, V.K. "Effects of accumulating work shifts on performance-based fatigue using multiple strength measurements in day and night shift nurses and aides". Hum Factors. 59: 346-356. 2017.

[17] Ferreira, T.S., Moreira, C.Z., Guo, J. and Noce, F. "Effects of a 12-hour shift on mood states and sleepiness of Neonatal Intensive Care Unit nurses". Rev Esc Enferm USP. 51: e03202. 2017.

[18] Karhula, K., Härmä, M., Sallinen, M., Hublin, C., Virkkala, J. and Kivimäki, M. "Job strain, sleep and alertness in shift working health care professionals -- a field study". Ind Health 51: 406-16. 2013.

[19] Kaliyaperumal, D., Elango, Y., Alagesan, M. and Santhanakrishanan, I. "Effects of sleep deprivation on the cognitive performance of nurses working in shift". J Clin Diagn Res. 11: CC01-CC03. 2017.

[20] Caruso, C.C., Baldwin, C.M., Berger, A., Chasens, E.R., Landis, C. and Redeker N.S. "Position statement: Reducing fatigue associated with sleep deficiency and work hours in nurses". Nurs Outlook. 65: 766-768. 2017.
[21] Vitale, S.A., Varrone-Ganesh, J. and Vu, M. "Nurses working the night shift: impact on home, family and social life". J Nurs Educ Pract 7:70-78. 2015.

[22] Ferri, P., Guadi, M., Marcheselli, L., Balduzzi, S., Magnani, D. and $\mathrm{Di}$ Lorenzo, R. "The impact of shift work on the psychological and physical health of nurses in a general hospital: a comparison between rotating night shifts and day shifts". Risk Manag Healthc Policy. 9: 203-211. 2016.

[23] Jensen, H.I., Larsen, J.W. and Thomsen, T.D. "The impact of shift work on intensive care nurses' lives outside work: a cross-sectional study". J Clin Nurs. 27: e703-e709. 2018.

[24] Barnes, M. Understanding participatory time for groups at risk of social exclusion. Economic and Social Research Council. 2011

[25] Jaradat, Y., Birkeland Nielsen, M., Kristensen, P. and BastPettersen, R. "Job satisfaction and mental health of Palestinian nurses with shift work: a cross-sectional study". Lancet. 391 Suppl 2: S50. 2018.

[26] Nasrabadi, A.N., Seif, H., Latifi, M., Rasoolzadeh, N. and Emami, A. "Night shift work experiences among Iranian nurses: a qualitative study". Int Nurs Rev 56: 498-503. 2009

[27] Yildirim, D. and Aycan, Z. "Nurses' work demands and work-family conflict: a questionnaire survey". Int J Nurs Stud. 45: 1366-1378. 2008.

[28] Almajwal, A.M. "Correlations of physical activity, body mass index, shift duty, and selected eating habits among nurses in Riyadh, Saudi Arabia". Ecol Food Nutr. 54: 397-417. 2015

[29] Lin, S.H., Liao, W.C., Chen, M.Y. and Fan, J.Y. "The impact of shift work on nurses' job stress, sleep quality and self-perceived health status". J Nurs Manag. 22: 604-612. 2014.

[30] Chen, C.H., Wang, J., Yang, C.S. and Fan, J.Y. "Nurse practitioner job content and stress effects on anxiety and depressive symptoms, and self-perceived health status". J Nurs Manag. 24: 695-704. 2016.

[31] Bohle, P. and Tilley, A.J. "The impact of night work on psychological well-being”. Ergonomics. 32: 1089-1099. 1989.

[32] Chen, M.J. and Weng, S.S. "Psychological symptoms among hospital nurses in Taiwan: a cross sectional study". BMC Women's Health. 17:101. 2017.

[33] Li, H., Shao, Y., Xing, Z., Li, Y., Wang, S. and Zhang, M. "Napping on night-shifts among nursing staff: A mixed-methods systematic review". J Adv Nurs. Review. 2018. 